\title{
Lithium abundances of main-sequence and subgiant stars in the globular cluster NGC 6397
}

\author{
J. I. González Hernández, ${ }^{1,2} \dagger$ P. Bonifacio, ${ }^{1,2,3}$ E. Caffau,${ }^{1}$ M. Steffen, ${ }^{4}$ \\ H.-G. Ludwig, ${ }^{1,2}$ N. Behara, ${ }^{1,2}$ L. Sbordone, ${ }^{1,2}$ R. Cayre $^{1}$ and \\ S. Zaggia ${ }^{5}$ \\ ${ }^{1}$ GEPI, Observatoire de Paris, CNRS, Université Paris Diderot, Place Jules Janssen, \\ 92190 Meudon, France \\ email: Jonay . Gonzalez-Hernandez@obspm .fr \\ ${ }^{2}$ Cosmological Impact of the First Stars (CIFIST) Marie Curie Excellence Team \\ ${ }^{3}$ Istituto Nazionale di Astrofisica, Observatorio Astronomico di Trieste, Italy \\ ${ }^{4}$ Astrophysikalisches Institut Potsdam, An der Sternwarte 16, D-14482 Potsdam, Germany \\ ${ }^{5}$ INAF, Osservatorio Astronomico di Padova, Vicolo dell'Osservatorio 5, Padua 35122, Italy
}

\begin{abstract}
We present FLAMES/GIRAFFE spectroscopy obtained with the Very Large Telescope (VLT). Using these observations, we have been able (for the first time) to observe the LiI doublet in the main-sequence (MS) stars of a globular cluster. We also observed Li in a sample of subgiant (SG) stars of the same $B-V$ colour. Our final sample is composed of $84 \mathrm{SG}$ and 79 MS stars. In spite of the fact that SG and MS stars span the same temperature range, we find that the equivalent widths of the LiI doublet in SG stars are systematically greater than in MS stars, suggesting a higher Li content among SG stars. This is confirmed by our quantitative analysis, which makes use of both $1 \mathrm{D}$ and $3 \mathrm{D}$ model atmospheres. We find that SG stars show, on average, a higher Li abundance, by $0.1 \mathrm{dex}$, than MS stars. We also detect a positive slope of $\mathrm{Li}$ abundance with effective temperature: the higher the temperature the higher the $\mathrm{Li}$ abundance, both for SG and MS stars, although the slope is slightly steeper for MS stars. These results provide unambiguous evidence that the $\mathrm{Li}$ abundance changes with evolutionary state. The physical mechanisms that contribute to this are not yet clear, since none of the proposed models seem to describe accurately the observations. Whether such a mechanism can explain the cosmological lithium problem is still an open question.
\end{abstract}

Keywords. stars: abundances, stars: atmospheres, stars: fundamental parameters, stars: Population II, globular clusters: individual (NGC 6397)

\section{Introduction}

The primordial Li abundance inferred from fluctuations in the cosmic-microwave background measured by the WMAP satellite (Spergel et al. 2007; Cyburt et al. 2008) is $\log (\mathrm{Li} / \mathrm{H})+12=2.72 \pm 0.06$ dex, approximately $0.3-0.5$ dex higher than the Li abundance determined in metal-poor stars in the Galactic halo.

Many studies have tried to explain this difference: (i) Piau et al. (2006) propose that the first generation of stars, Population III stars, could have processed some fraction of the halo gas, thus lowering the lithium abundance, while (ii) other authors suggest that the primordial Li abundance has been uniformly depleted in the atmospheres of metal-poor dwarfs by some physical mechanism (e.g., turbulent diffusion, as in Richard

$\dagger$ Present address: Dpto. de Astrofísica y Ciencias de la Atmósfera, Facultad de Física, Universidad Complutense de Madrid, E-28040 Madrid, Spain, email: jonay@astrax.fis.ucm.es 
et al. 2005 and Korn et al. 2006; gravitational waves, as in Charbonnel \& Talon 2005, etc.). (iii) Finally, it has also been suggested that the standard Big Bang nucleosynthesis (SBBN) calculations should be revised, possibly by introducing new physics as in, e.g., Jedamzik (2004), Jedamzik (2006), Jittoh et al. (2008) and Hisano et al. (2009).

Here, we present the Li abundances of subgiant (SG) and main-sequence (MS) stars in NGC 6397. This study represents the first Li-abundance measurements in MS stars in a globular cluster.

\section{Observations}

Spectroscopic observations of the globular cluster NGC 6397 were carried out with the multi-object spectrograph FLAMES/GIRAFFE at the VLT in 2007 April, May, June and July, covering the spectral range $\lambda \lambda 6400-6800 \AA$ at a resolving power of $\lambda / \delta \lambda \sim 17000$.

We selected subgiant and dwarf stars in the colour range $B-V=0.6 \pm 0.03 \mathrm{mag}$, which ensures that both sets of stars fall in a similar and narrow effective-temperature range (see figure 3 online in González Hernández et al. 2009).

The spectra were reduced with the ESO pipeline and later on treated within MIDAS. We corrected the spectra for sky lines and barycentric and radial velocities. We typically combine 17 spectra of dwarfs and 4 spectra of subgiants to achieve a similar signal-tonoise ratio. The mean radial velocity of the cluster stars is $v_{\mathrm{r}}=18.5 \mathrm{~km} \mathrm{~s}^{-1}$.

\section{Stellar parameters}

We derived the effective temperature by fitting the observed $\mathrm{H} \alpha$ line profiles with synthetic profiles, using $3 \mathrm{D}$ hydrodynamical model atmospheres computed with the $\mathrm{CO}^{5} \mathrm{BOLD}$ code (Freytag et al. 2002; Wedemeyer et al. 2004). The ability of 3D models to reproduce Balmer-line profiles has been shown by Behara et al. (2009), where the H $\alpha$ profiles of the Sun and the metal-poor stars HD 84937, HD 74000 and HD 140283 were investigated. See Ludwig et al. (2009) for further details.

The effective temperatures of MS and SG stars were also derived adopting 1D ATLAS 9 model atmospheres (see Kurucz 2005) and using the same fitting procedure.

Fixed values for the surface gravity were adopted for both subgiant and dwarf stars, according to the values that best match the position of the stars on a 12 Gyr isochrone (Straniero et al. 1997). The adopted values were $\log \left(\mathrm{g} / \mathrm{cm} \mathrm{s}^{2}\right)=4.40$ and 3.85 for MS and SG stars, respectively.

\section{Li abundances}

We measure the equivalent width (EW) of the LiI $6708 \AA$ line in SG and MS stars by fitting synthetic spectra of known EW to the observed Li profiles. The SG stars show on average larger EWs than MS stars. This is clearly seen in figure 1 of González Hernández et al. (2009), where the histograms of the EWs measured in SG and MS stars in this cluster are displayed. Although the $B-V$ colour is sensitive to surface gravity, this result was not expected a priori and suggests that subgiants in this cluster have actually higher Li abundances than dwarfs.

The $\mathrm{Li}$ abundances were derived using 3D model atmospheres. Line formation of $\mathrm{Li}$ was treated in nonlocal thermodynamical equilibrium (NLTE) using the same code and model atoms used in Cayrel et al. (2007). The analysis was also done with 1D model atmospheres, providing essentially the same picture, although $T_{\text {eff }}$ in $1 \mathrm{D}$ yields lower 


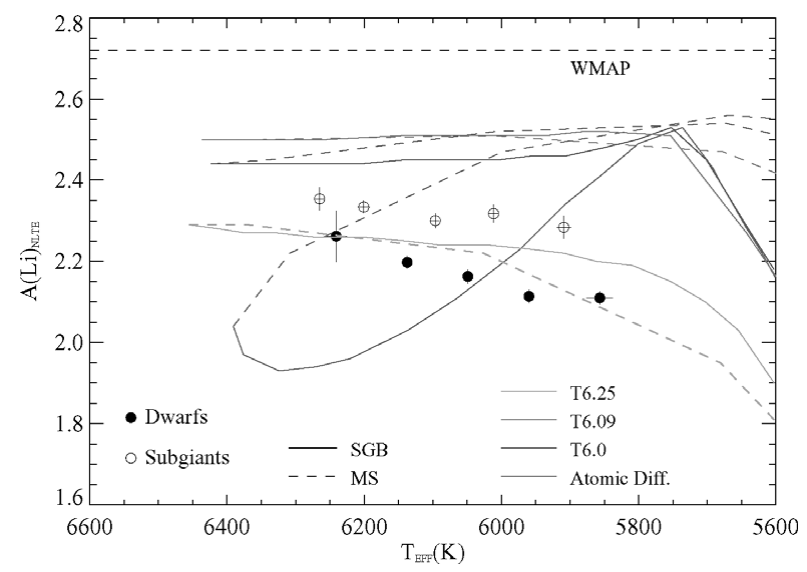

Figure 1. 1D NLTE (nonlocal thermodynamical equilibrium) Li abundances versus 1D effective temperatures of the observed stars and $\mathrm{Li}$ isochrones for different turbulent diffusion models. Black solid and open circles correspond to dwarf and subgiant stars, respectively. Different turbulent diffusion model isochrones of lithium are displayed for different levels of turbulence. In each isochrone, the dashed section of the line shows the $\mathrm{Li}$ abundance in dwarf stars, while the solid line shows that in subgiant stars. The horizontal black dashed line represents the cosmological Li abundance.

values (see figure 6 online in González Hernández et al. 2009). In the $1 \mathrm{D}$ case, we use the Carlsson et al. (1994) NLTE corrections.

\section{Discussion and conclusions}

In Figure 1 we display the derived 1D-NLTE Li abundances versus the 1D effective temperatures of dwarf and subgiant stars in the globular cluster NGC 6397 [see figure 2 in González Hernández et al. (2009) for a similar picture but with $T_{\text {eff }}$ and Li abundances computed using 3D models]. The stars have been divided into five bins of effective temperature. The error bar in the Li abundance is the dispersion for each bin, divided by the square root of the number of stars in the bin. The Li abundance decreases with decreasing temperature. This lithium-abundance pattern is different from that found among field stars (Meléndez \& Ramírez 2004; Bonifacio et al. 2007; González Hernández et al. 2008).

The difference in the $\mathrm{Li}$ abundance of dwarfs and subgiants is $\sim 0.14$ dex if one computes the mean $1 \mathrm{D} A(\mathrm{Li})$ and the standard deviation of the mean for the two samples. For subgiants and dwarfs we find mean 1D Li abundances of $2.31 \pm 0.01$ and $2.17 \pm 0.01$ dex, respectively. The 3D models provide similar results, although slightly higher Li abundances. Lind et al. (2009) also find such differences between the mean Li abundances in MSs and SGs, but only by 0.03 dex, although this is still signicant at $1 \sigma$. However, this result is partly affected by the very narrow range of $T_{\text {eff }}$ for MS stars derived by Lind et al. $(2009)(\sim 80 \mathrm{~K})$ compared to the wide range $(\sim 450 \mathrm{~K})$ for the SGs (see figure 7 online in González Hernández et al. 2009).

Our results imply that the Li surface abundance depends on the evolutionary status of the star. In Figure 1 we show the Li isochrones for different turbulent-diffusion models (Richard et al. 2005). These models have been shifted up by 0.14 dex in Li abundance to make the initial abundance of the models, $\log (\mathrm{Li} / \mathrm{H})=2.58 \mathrm{dex}$, coincide with the primordial Li abundance predicted from fluctuations of the microwave background measured by the WMAP satellite (Cyburt et al. 2008). The models assuming pure atomic diffusion, and, among those including turbulent mixing, T6.0 and T6.09, are ruled out 
by our observations. All such models predict that in dwarf stars Li should be either more abundant or the same as in subgiant stars. The only model that predicts a Li pattern which is qualitatively similar to that observed is the T6.25 model.

Models including atomic diffusion and tachocline mixing (Piau 2008) do not seem to reproduce our observations, since they provide a constant $\mathrm{Li}$ abundance up to $5500 \mathrm{~K}$. The sophisticated models which, in addition to diffusion and rotation, also take into account the effects of internal gravity waves (Talon \& Charbonnel 2004) seem to predict accurately the Li abundance pattern in solar-type stars, at solar metallicity (Charbonnel \& Talon 2005), but models at low metallicity are still needed.

The cosmological lithium discrepancy still needs to be solved. Given that none of the existing models of Li evolution in stellar atmospheres match the observations, we hope our results will prompt further new theoretical investigations.

\section{References}

Behara, N. T., Ludwig, H.-G., Steffen, M., \& Bonifacio, P. 2009, AIP Conf. Ser., 1094, 784

Bonifacio, P., et al. 2007, A\& A, 462, 851

Carlsson, M., Rutten, R. J., Bruls, J. H. M. J., \& Shchukina, N. G. 1994, A\&\&A, 288, 860

Cayrel, R., Steffen, M., Chand, H., Bonifacio, P., Spite, M., Spite, F., Petitjean, P., Ludwig, H.-G., \& Caffau, E. 2007, $A \mathscr{B} A$ (Letters), 473, L37

Charbonnel, C. \& Talon, S. 2005, Science, 309, 2189

Cyburt, R. H., Fields, B. D., \& Olive, K. A. 2008, J. Cosmol. Astro-Part. Phys., 11, 12

Freytag, B., Steffen, M., \& Dorch, B. 2002, AN, 323, 213

González Hernández, J. I., et al. 2008, A\&̈A, 480, 233

González Hernández, J. I., et al. 2009, A\&\&A (Letters), 505, L13

Hisano, J., Kawasaki, M., Kohri, K., \& Nakayama, K. 2009, Phys. Rev. D, 79, 063514

Jedamzik, K. 2004, Phys. Rev. D, 70, 083510

Jedamzik, K. 2006, Phys. Rev. D, 74, 103509

Jittoh, T., Kohri, K., Koike, M., Sato, J., Shimomura, T., \& Yamanaka, M. 2008, Phys. Rev. $D, 78,055007$

Kurucz, R. L. 2005, Mem. Soc. Astron. Ital. Suppl., 8, 14

Korn, A. J., Grundahl, F., Richard, O., Barklem, P. S., Mashonkina, L., Collet, R., Piskunov, N., \& Gustafsson, B. 2006, Nature, 442, 657

Lind, K., Primas, F., Charbonnel, C., Grundahl, F., \& Asplund, M. 2009, A $\& A, ~ 503,545$

Ludwig, H.-G., Behara, N. T., Steffen, M., \& Bonifacio, P. 2009, A $\mathscr{E} A$ (Letters), 502, L1

Meléndez, J. \& Ramírez, I. 2004, ApJ (Letters), 615, L33

Piau, L., Beers, T. C., Balsara, D. S., Sivarani, T., Truran, J. W., \& Ferguson, J. W. 2006, ApJ, 653, 300

Piau, L. 2008, ApJ, 689, 1279

Richard, O., Michaud, G., \& Richer, J. 2005, ApJ, 619, 538

Spergel, D. N., et al. 2007, ApJS, 170, 377

Straniero, O., Chieffi, A., \& Limongi, M. 1997, ApJ, 490, 425

Talon, S. \& Charbonnel, C. 2004, A\&A, 418, 1051

Wedemeyer, S., Freytag, B., Steffen, M., Ludwig, H.-G., \& Holweger, H. 2004, A\&SA, 414, 1121 